\title{
Atmospheric Soundings from AIRS/AMSU in Partial Cloud Cover
}

\author{
Joel Susskind and Robert Atlas \\ NASA Goddard Space Flight Center, Greenbelt, MD, USA 20771
}

\begin{abstract}
Simultaneous use of AIRS/AMSU-A observations allow for the determination of accurate atmospheric soundings under partial cloud cover conditions. The methodology involves the determination of the radiances AIRS would have seen if the AIRS fields of view were clear, called clear column radiances, and use of these radiances to infer the atmospheric and surface conditions giving rise to these clear column radiances. Susskind et al. ${ }^{1}$ demonstrate via simulation that accurate temperature soundings and clear column radiances can be derived from AIRS/AMSU-A observations in cases of up to $80 \%$ partial cloud cover, with only a small degradation in accuracy compared to that obtained in clear scenes. Susskind and Atlas $^{2}$ show that these findings hold for real AIRS/AMSU-A soundings as well. For data assimilation purposes, this small degradation in accuracy is more than offset by a significant increase in spatial coverage (roughly $50 \%$ of global cases were accepted, compared to $3.6 \%$ of the global cases being diagnosed as clear), and assimilation of AIRS temperature soundings in partially cloudy conditions resulted in a larger improvement in forecast skill than when AIRS soundings were assimilated only under clear conditions. Altematively, derived AIRS clear column radiances under partial cloud cover could also be used for data assimilation purposes. Further improvements in AIRS sounding methodology have been made since the results shown in Susskind and Atlas ${ }^{2}$. A new version of the AIRS/AMSU-A retrieval algorithm, Version 4.0, was delivered to the Goddard DAAC in February 2005 for production of AIRS derived products, including clear column radiances. The major improvement in the Version 4.0 retrieval algorithm is with regard to a more flexible, parameter dependent, quality control. Results are shown of the accuracy and spatial distribution of temperature-moisture profiles and clear column radiances derived from AIRS/AMSU-A as a function of fractional cloud cover using the Version 4.0 algorithm. Use of the Version 4.0 AIRS temperature profiles increased the positive forecast impact arising from AIRS retrievals relative to what was shown in Susskind and Atlas ${ }^{2}$.
\end{abstract}

Keywords: infra-red, microwave, remote sensing, temperature, moisture, high spectral resolution, clouds, forecast, meteorology

\section{INTRODUCTION}

AIRS/AMSU/HSB is a state of the art advanced infra-red microwave sounding system that was launched on the EOS Aqua platform in a 1:30 AM/PM sun synchronous orbit on May 4, 2002. An overview of the AIRS instrument is given in Pagano et $\mathrm{al}^{3}$. The sounding goals of AIRS are to produce $1 \mathrm{~km}$ tropospheric layer mean temperatures with an $\mathrm{mms}$ error of $1 \mathrm{~K}$, and layer precipitable water with an rms error of $20 \%$, in cases with up to $80 \%$ effective cloud cover. Aside from being part of a climate mission, one of the objectives of AIRS is to provide sounding information of sufficient accuracy such that when assimilated into a general circulation model, significant improvement in forecast skill would arise. The pre-launch algorithm to produce level 2 products (geophysical parameters) using AIRS/AMSU/HSB data, and expected results based on simulation studies, are given in Susskind et al. ${ }^{1}$ The results of that simulation indicate that the sounding goals of AIRS/AMSU/HSB should be achievable. In that simulation, perfect knowledge of the instrumental spectral response functions and the inherent physics of the radiative transfer equations were assumed. Therefore, if the true state of the atmosphere and underlying surface were known perfectly, one could compute the radiances AIRS, AMSU, and HSB would see exactly up to instrumental noise. Susskind et al. ${ }^{1}$ alluded to the fact that this is not the case in reality, and additional terms would have to be included in the retrieval algorithm to account for systematic differences (biases) between observed brightness temperatures and those computed knowing the "true" surface and atmospheric state, as well as for residual computational errors after that systematic bias is accounted for (computational noise). In this paper, we show results based on the algorithm we were using to analyze AIRS/AMSU/HSB data on November 30, 2004, which we will refer to as Version 4.0. This algorithm is very similar to the pre-launch version, with the major differences attributable to the factors described above. In addition, we now have separate quality indicators for different 
geophysical parameters, as opposed to the entire state being flagged as good or bad. JPL delivered an earlier version of the algorithm, Version 3.0, to the Goddard DAAC, for the earliest near real time processing of AIRS level 2 products starting in August 2003. We have used Version 4.0 to analyze data for the AIRS focus day September 6, 2002, and all of January 2003 and January 2004 for use in forecast impact experiments and a study of interannual monthly mean differences. Research to further improve the results of analysis of AIRS/AMSU/HSB data is continuing. The Goddard DAAC will begin analyzing AIRS/AMSU data using the Version 4.0 retrieval algorithm, in early 2005 , to process near real time AIRS data from that point forward, as well as reprocess all AIRS data from September 2002, when the instrument became stable.

\section{OVERVIEW OF THE AIRS TEAM RETRIEVAL ALGORITHM}

The AIRS team retrieval algorithm is basically identical to that described in Susskind et al. ${ }^{1 .}$ The key steps are outlined below: 1) Start with an initial state consistent with the AMSU A and HSB radiances ${ }^{4}$; 2) Derive IR clear column radiances $\hat{R}_{i}^{0}$ valid for the $3 \times 3$ AIRS Fields of View (FOVs) within an AMSU A Field of Regard (FOR) consistent with the observed radiances and the initial state; 3) Obtain an AIRS regression guess ${ }^{5}$ consistent with $\hat{R}_{i}^{0}$ using 1504 AIRS channels; 4) Derive $\hat{R}_{\mathbf{i}}^{1}$ consistent with the AIRS radiances and the regression guess; 5) Derive all surface and atmospheric parameters using $\hat{R}_{\hat{i}}^{1}$ for 293 AIRS channels and all AMSU radiances; 6) Derive cloud parameters and OLR consistent with the solution and observed $R_{i}$; 7) Apply initial quality control, which rejects a solution if the retrieved cloud fraction is greater than $90 \%$ or other relatively coarse tests fail. In the event that a retrieval is rejected initially, cloud parameters are determined consistent with the initial microwave state and observed AIRS radiances. Otherwise, cloud parameters are computed based on the coupled IR/MW retrieval, and further quality control is applied to individual geophysical parameters.

\section{QUALITY CONTROL}

Coupled AIRS/AMSU retrievals in the presence of broken cloud cover are usually highly accurate. Under some conditions, such as complete overcast, combined AIRS/AMSU retrievals cannot be performed at all. In cases of complex clouds or terrain, retrievals are of poorer quality. In earlier versions of the AIRS/AMSU retrieval algorithm, quality control was applied uniformly to the entire profile. If any geophysical parameter was considered to be of poor quality, the whole set of geophysical parameters was rejected and clouds were derived using the microwave initial state in Step (1) above. This "one size fits all" approach led to significant compromises between desired spatial coverage of accepted retrievals and desired accuracy. Now, we report the combined IR/MW retrieval parameters, and use them to derive cloud parameters, as long as we feel the combined IR/MW retrieval (Step 5) is at least as accurate as the MW only retrieval (Step 1). This is considered to be true if the retrieved cloud fraction derived using the IRMW state is less than or equal to $90 \%$ and the initial cloud clearing step was stable. If this test is passed (the Stratospheric Temperature Test), the temperature profile above $200 \mathrm{mb}$ is considered acceptable. Constituent profiles $\left(\mathrm{H}_{2} \mathrm{O}, \mathrm{O}_{3}, \mathrm{CO}\right.$, and $\left.\mathrm{CH}_{4}\right)$ are accepted if the Stratospheric Temperature Test is passed and additional slightly more stringent cloud clearing stability tests are also passed. Only a few percent of the cases passing the Stratospheric Temperature Test are not accepted for constituent profile. The next level of test is applied to the temperature profile beneath $200 \mathrm{mb}$ and above $3 \mathrm{~km}$ (the Mid Tropospheric Temperature Test). Finally, a more stringent test is applied to accept temperature profiles in the lowest 3 $\mathbf{k m}$ of the atmosphere (Lower Tropospheric Temperature Test). Lower tropospheric temperatures are the most difficult to determine accurately, both because of effects of low clouds on the radiances and uncertainty and small scale variability in surface skin temperature and emissivity. Both concerns create greater problems over land than ocean. In response to this, the Lower Tropospheric Temperature Test rejects lower tropospheric temperature more often over land than over ocean.

Temperatures flagged as accepted are candidates for use in data assimilation, for which high accuracy is required. If the same tight acceptance criteria were used when generating monthly mean temperature data sets at different levels of the atmosphere, gaps in low level temperatures would exist over certain land areas, primarily deserts during the day. For this reason, we include low level temperatures in the generation of monthly mean fields for all soundings which pass the Mid-Tropospheric Temperature Test. For the same reason, this test is also applied when generating land surface skin temperature data sets, for which other good information does not exist, and the highest accuracy is not required. The 
same is not true for ocean skin temperature, as accurate ocean skin temperatures are produced routinely using IR imagery data such as AVHRR or MODIS. Therefore, for ocean skin temperatures, the rejection thresholds are further tightened from those accepted temperatures in the lowest $3 \mathrm{~km}$, with a goal of less than $1 \%$ outliers of sea surface temperatures compared to other measurements. We currently have two sets of SST thresholds, trading off between accuracy and coverage, which we will refer to as the Tight SST Test and the Loose SST Test.

\section{RESULTS USING VERSION 4.0}

Figure 1a shows the retrieved effective cloud top pressure and effective cloud fraction for ascending orbits on September 6,2002 . The global mean effective cloud fraction and its spatial standard deviation are indicated in the figure. The results are presented in terms of cloud fraction in 5 groups, $0-20 \%, 20-40 \%$, etc. with darker colors indicating greater cloud cover. These groups are shown in each of 7 colors, indicative of cloud top pressure. The reds and purples indicate the highest clouds, and the yellows and oranges the lowest clouds. Cloud fields are retrieved for all cases in which valid AIRS/AMSU observations exist. Gray means no data was observed. Figure $1 \mathrm{~b}$ shows the retrieved $200 \mathrm{mb}$ temperature field. This demonstrates the coverage of cases where stratospheric temperatures are accepted. Gray indicates regions where either no valid observations existed or the stratospheric temperature retrieval was rejected, generally in regions of cloud cover $90-100 \%$. Figure 1c shows retrieved values of total precipitable water vapor above the surface. This demonstrates the coverage of constituent profiles. Figure $1 \mathrm{~d}$ shows results of $500 \mathrm{mb}$ temperature, demonstrating coverage of mid-tropospheric temperatures. Gaps in the data coverage of mid-tropospheric temperature due to extensive cloud cover are larger than for stratospheric temperatures. Retrieved fields are quite coherent, and show no apparent artifacts due to clouds in the field of view. Water vapor has considerably more fine scale structure than temperature and contains some very large spatial gradients.

\section{September 60 P. 2002}
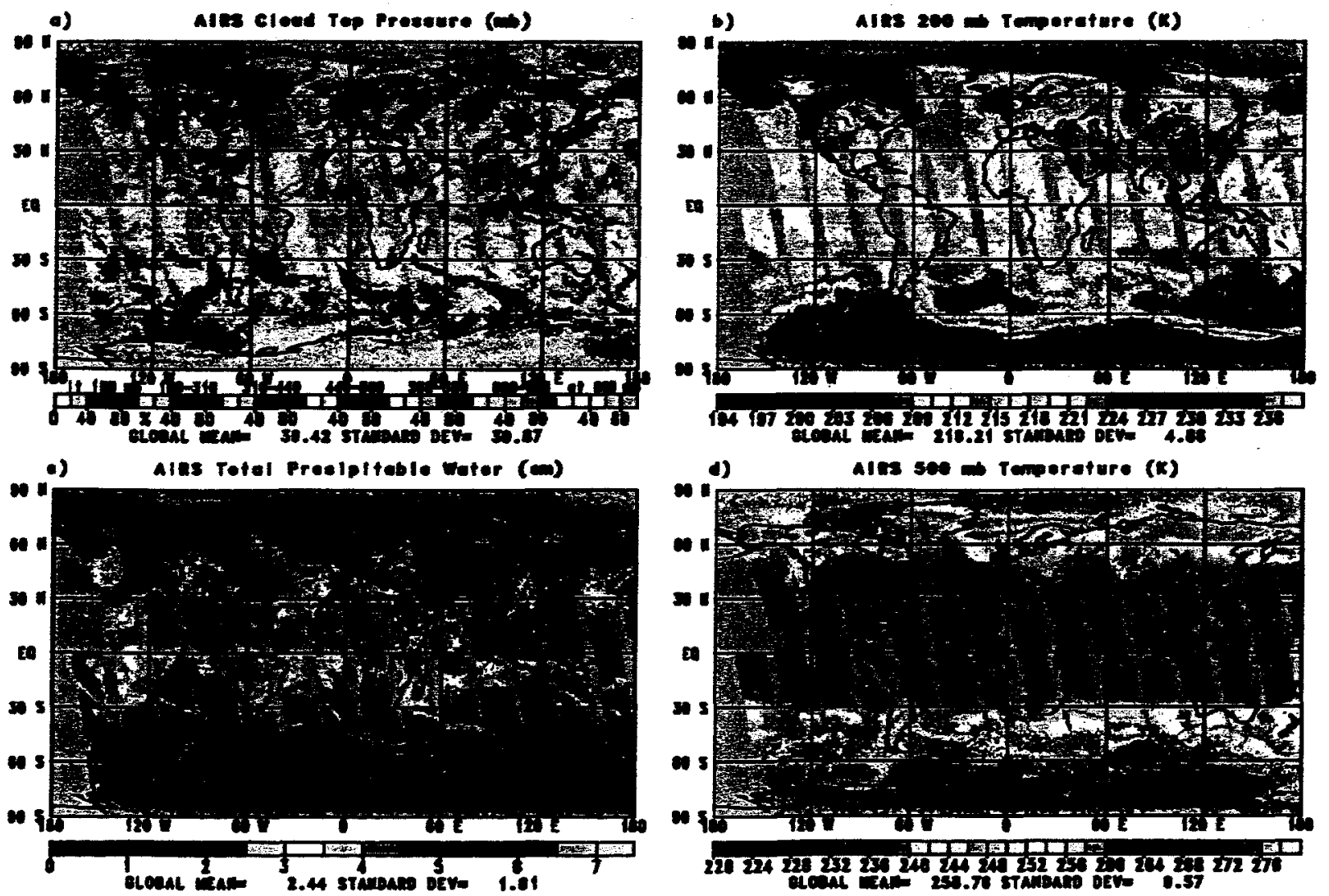

Figure 1 
Figure 2 shows the number of cases for each retrieved effective fractional cloud cover, in $0.5 \%$ bins, for the whole day September 6, 2002. The effective fractional cloud cover is given by the product of the fraction of the field of view covered by clouds and the cloud emissivity at $11 \mu \mathrm{m}$. The average global effective cloudiness was determined to be 42.61\%. Also shown is the percent of accepted retrievals as a function of retrieved effective cloud cover for all cases passing the Stratospheric Temperature Test, the Mid-Tropospheric Temperature Test, and the Lower Tropospheric Temperature Test, as well as non-frozen ocean cases passing the Loose SST Test and the Tight SST Test. Almost all cases with retrieved effective cloud fraction less than $90 \%$ pass the Stratospheric Temperature Test, with the percent yield falling slowly with increasing cloud cover, from close to $100 \%$ at low cloud fractions to about $65 \%$ at close to $90 \%$ cloud cover. $82 \%$ of the global cases pass the Stratospheric Temperature Test, with an average effective cloud fraction of $32.95 \%$. $52 \%$ of the global cases pass the Mid-Troposphere Temperature Test, with an acceptance rate of about $82 \%$ for low effective cloud fraction, falling to about $20 \%$ at $80 \%$ effective cloud fraction, and $10 \%$ at $90 \%$ effective cloud fraction. The previous acceptance methodology ${ }^{1}$ rejected all cases with effective cloud fraction greater than $80 \%$. The mean effective cloud fraction for all cases passing the Mid-Tropospheric Temperature Test is $25.25 \%$. Only $29 \%$ of the cases pass the Lower Tropospheric Temperature Test, primarily over ocean, with an acceptance rate near $55 \%$ for low cloud fractions falling to $7 \%$ at $80 \%$ effective cloud fraction and $3 \%$ at $90 \%$ effective cloud fraction, and an average effective cloud fraction of $20.40 \%$. The SST acceptance tests are applied only over non-frozen ocean. The Loose SST Test accepts about $21 \%$ of the ocean cases, with an acceptance rate of $50 \%$ under nearly clear conditions, and an average cloud fraction of $10.14 \%$, while the tight SST Test accepts only $9 \%$ of the cases, with an average effective cloud fraction of $7.05 \%$.

Percent Accepted vs. Effective Cloud Fraction

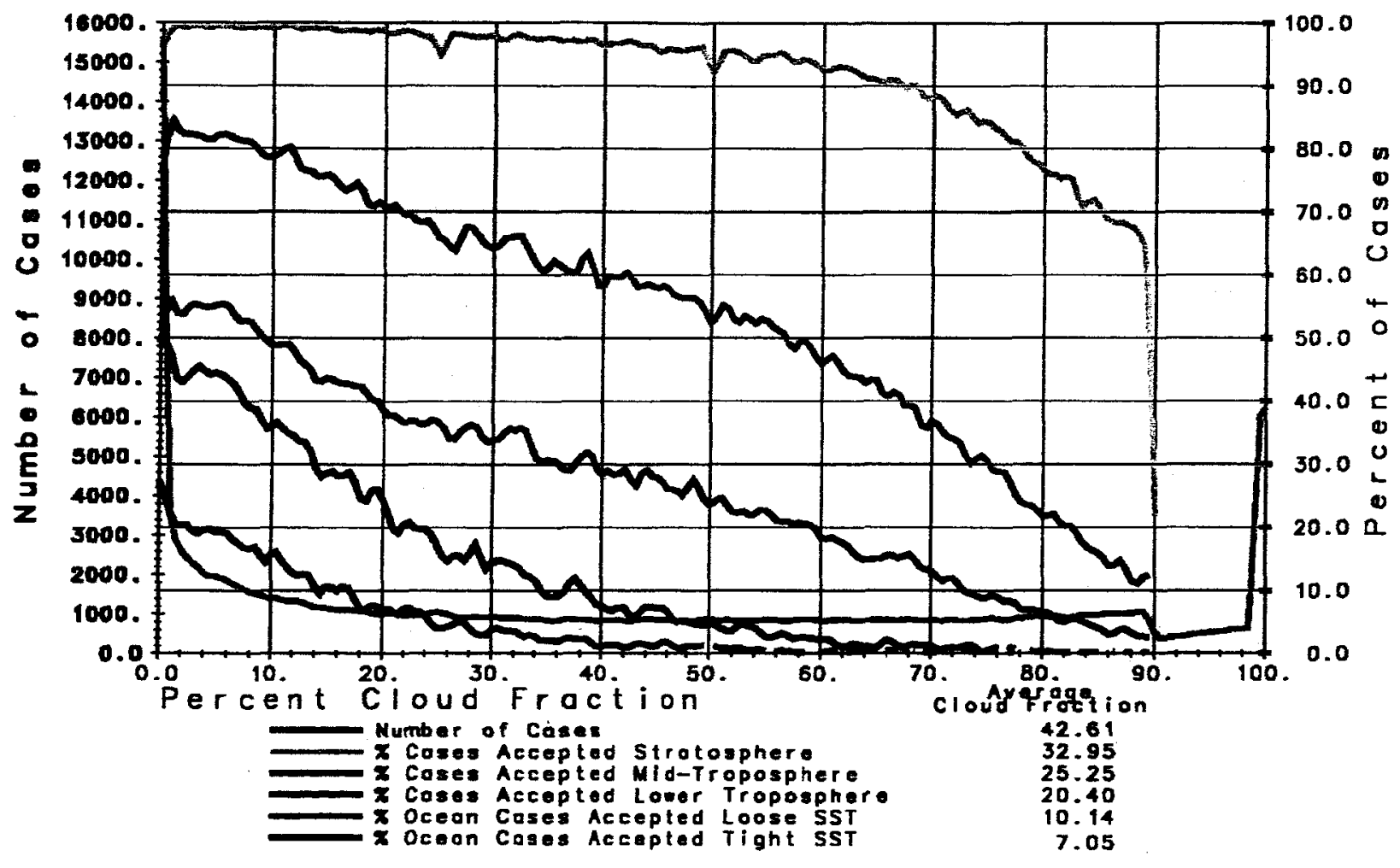

Figure 2 
Figure 3a shows the difference between the retrieved $700 \mathrm{mb}$ temperature and the ECMWF 6 hour forecast field for ascending orbits on September 6, 2002, for those cases passing the Lower Tropospheric Temperature Test, while Figure $3 \mathrm{~b}$ shows the same field for all cases passing the looser Mid-Tropospheric Temperature Test. The difference in spatial coverage is significant, particularly over land where $700 \mathrm{mb}$ temperature retrievals appear to be biased warm compared to the ECMWF forecast. The overall accuracy is somewhat better with the tighter acceptance criteria, and this difference is significant for data assimilation purposes. When statistics are shown depicting the accuracy of lower tropospheric temperatures (Figures 5 and 6), only cases passing the Lower Tropospheric Temperature Test are included. All data shown in Figure $3 \mathrm{~b}$ is included in the generation of monthly mean fields however.

Figures $3 \mathrm{c}$ and $3 \mathrm{~d}$ shows the differences of retrieved non-frozen ocean surface skin temperature (SST) from the ECMWF SST analysis for the ascending orbits of September 6,2002. Figure 3c includes only those cases passing the Tight SST test and Figure 3d also includes those cases passing the Loose SST Test. A considerable increase in yield is obtained using the Loose SST Test, with some degradation in accuracy of Sea Surface Temperatures.

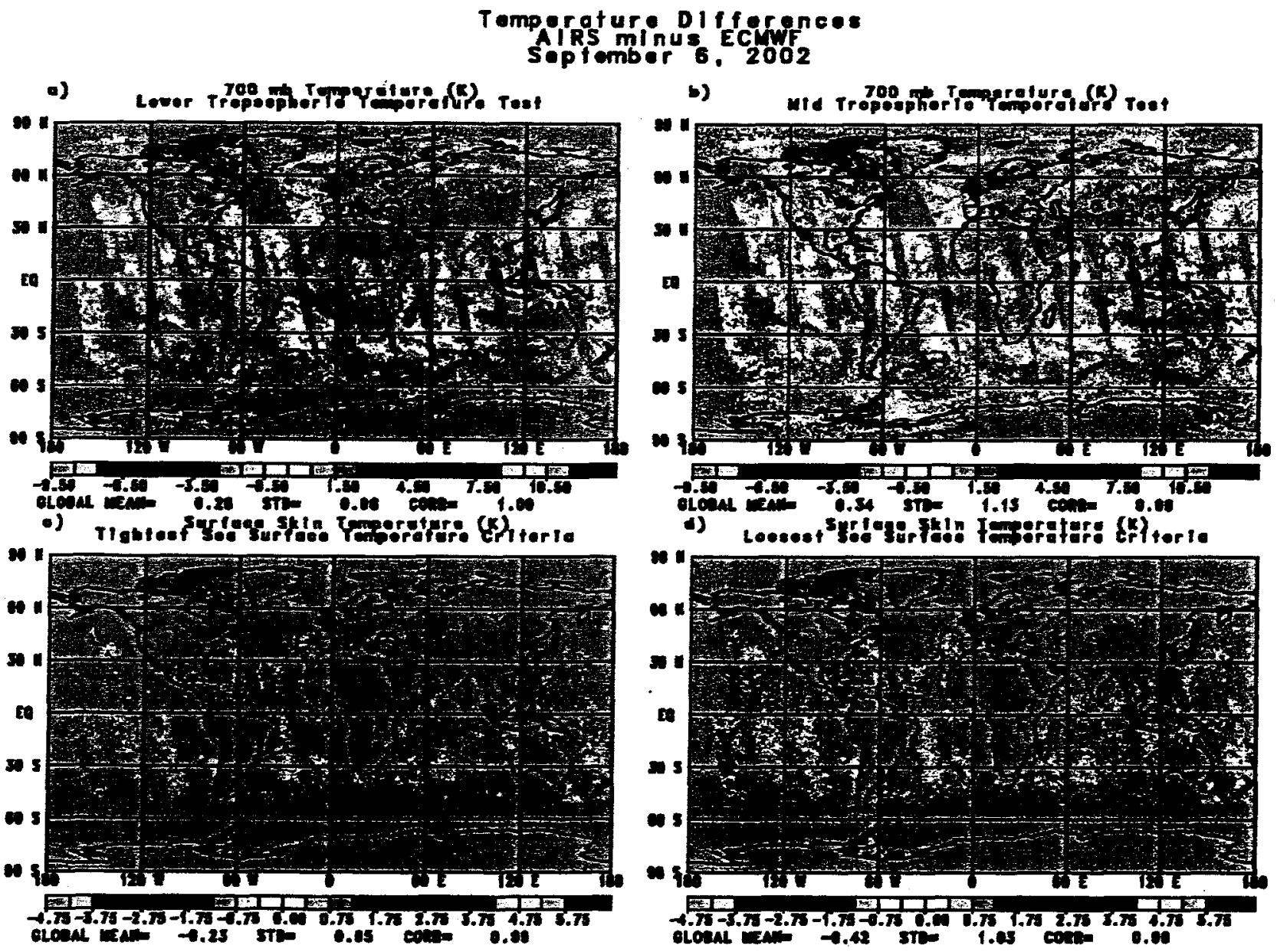

Figure 3 
Figure 4 shows the number of daytime and nighttime non-frozen ocean cases between $50^{\circ} \mathrm{N}$ and $50^{\circ} \mathrm{S}$, on September 6 , 2002 , as a function of the difference of the retrieved SST from the ECMWF analysis. Results are shown for cases which passed the Tight SST Test, the Loose SST Test, and the Lower Tropospheric Temperature Test. Figure 3c and 3d showed the spatial distribution differences for the daytime orbits appiying each of the SST Tests. Figure 4 includes statistics compared to ECMWF showing the percent of all non-frozen oceanic cases $50^{\circ} \mathrm{N}-50^{\circ} \mathrm{S}$ included in the statistics, the mean difference, the standard deviation of the difference, and the percentage of outliers, defined as cases passing the test that differ from ECMWF by more than 3K. There is a small negative bias of retrieved Sea Surface Temperatures compared to ECMWF, that increases with increasing acceptance rate, from $-0.21 \mathrm{~K}$ for cases within the Tight SST Test, to $-0.76 \mathrm{~K}$ for cases passing the Lower Tropospheric Temperature Test. The standard deviation of the cases from ECMWF also increases slightly. On the other hand, the number of primarily cold outliers increases significantly, from $0.68 \%$ to $7.28 \%$. Therefore the Lower Tropospheric Temperature Test by itself is not adequate for the purpose of producing accurate monthly mean sea surface temperatures.
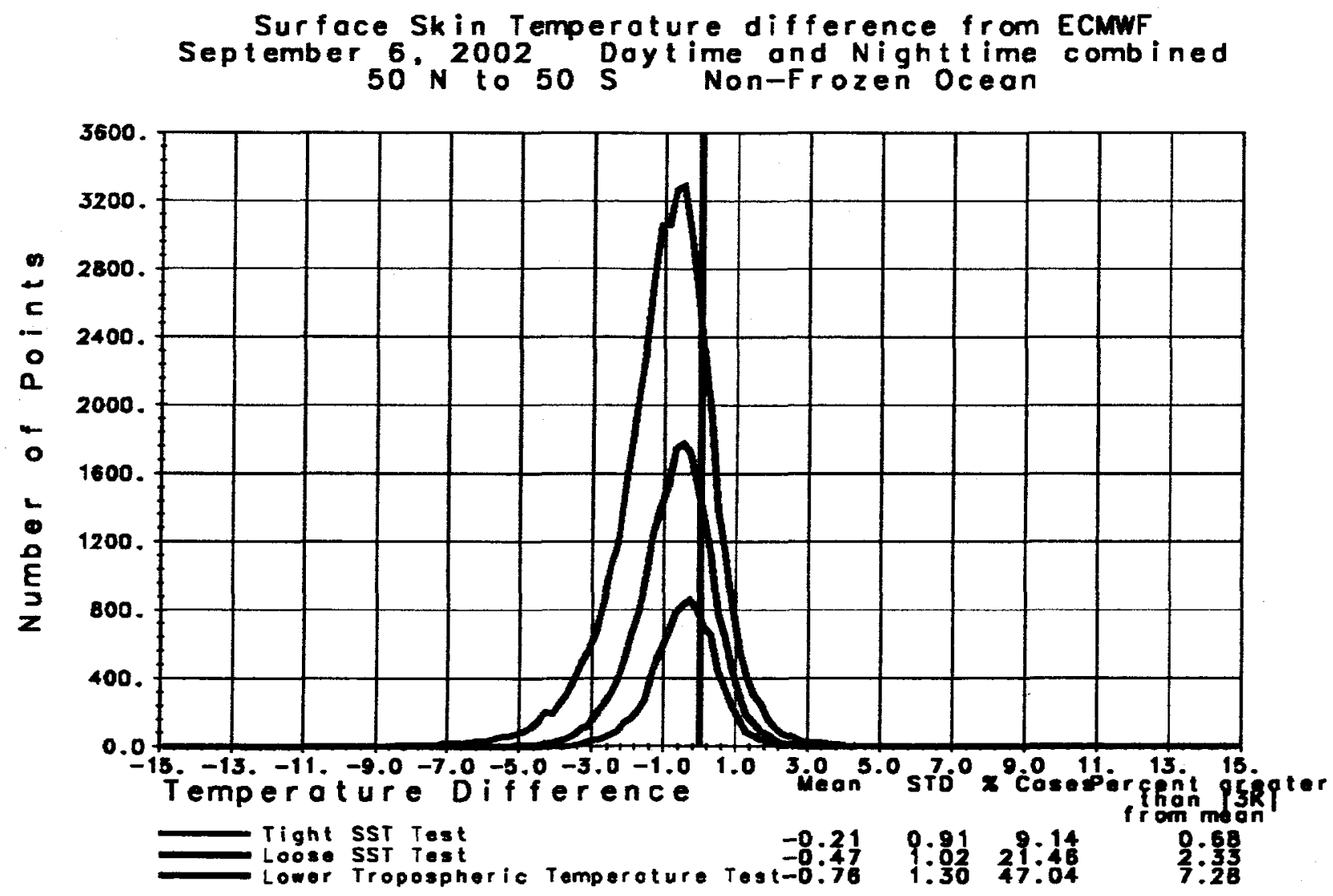

Figure 4

Figure 5a shows RMS differences from the ECMWF forecast of retrieved $1 \mathrm{~km}$ layer mean tropospheric temperatures, and $3 \mathrm{~km}$ layer mean stratospheric temperatures, for non-frozen ocean cases on September 6, 2002. Results shown are for all cases passing the Stratospheric Temperature Test, the cases passing the Mid-Tropospheric Test the Lower Tropospheric Temperature Test, the Loose SST Test, and the Tight SST Test. The number of cases and percentage of all cases included in the mid-tropospheric and lower-tropospheric statistics is included for each test.

Differences of retrieved stratospheric temperature from ECMWF are not appreciably different from one another for cases passing any of the quality flags. The large differences from ECMWF above $15 \mathrm{mb}$ are primarily a result of the lower accuracy of the ECMWF "truth" in the upper stratosphere. Tropospheric soundings passing the tropospheric quality control tests agree with the ECMWF forecast on the order of $1 \mathrm{~K}$. Part of this difference is due to uncertainty in the 
ECMWF forecast. It is interesting to note that soundings for the $87 \%$ of the cases for which the Stratospheric Temperature Test was passed are of high quality throughout the troposphere as well, with an RMS difference from ECMWF on the order of $1.7 \mathrm{~K}$ in the lowest $1 \mathrm{~km}$ of the atmosphere. This shows that the cloud clearing methodology works wcll in up to $90 \%$ cloud cover. Nevertheless, the accuracy of these soundings is not considered high enough for either data assimilation or climate purposes. There is further improvement in tropospheric temperature profile accuracy using the smaller subset of cases passing the Loose SST Test (21.45\% of the ocean cases) but relatively little further improvement in those cases passing the Tight SST Test (9.12\% of the cases).

Figure $5 \mathrm{~b}$ shows analogous results for global accepted retrievals, including cases passing the Stratospheric Temperature Test and the Mid and Lower Tropospheric Temperature Tests. Error statistics in the stratosphere are again similar for stratospheric cases $(82.52 \%)$ and tropospheric accepted cases $(52.51 \%)$. Global agreement with ECMWF is slightly poorer than over non-frozen ocean.

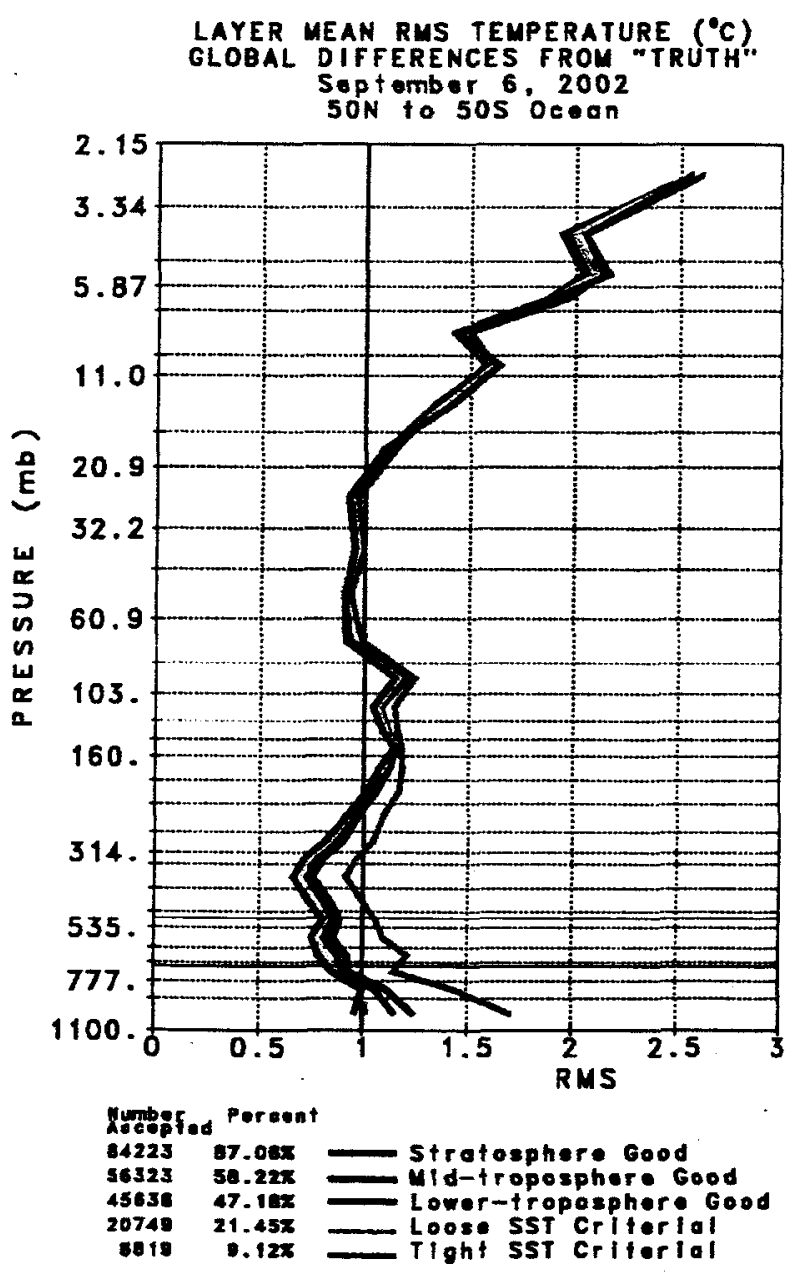

Figure 5a

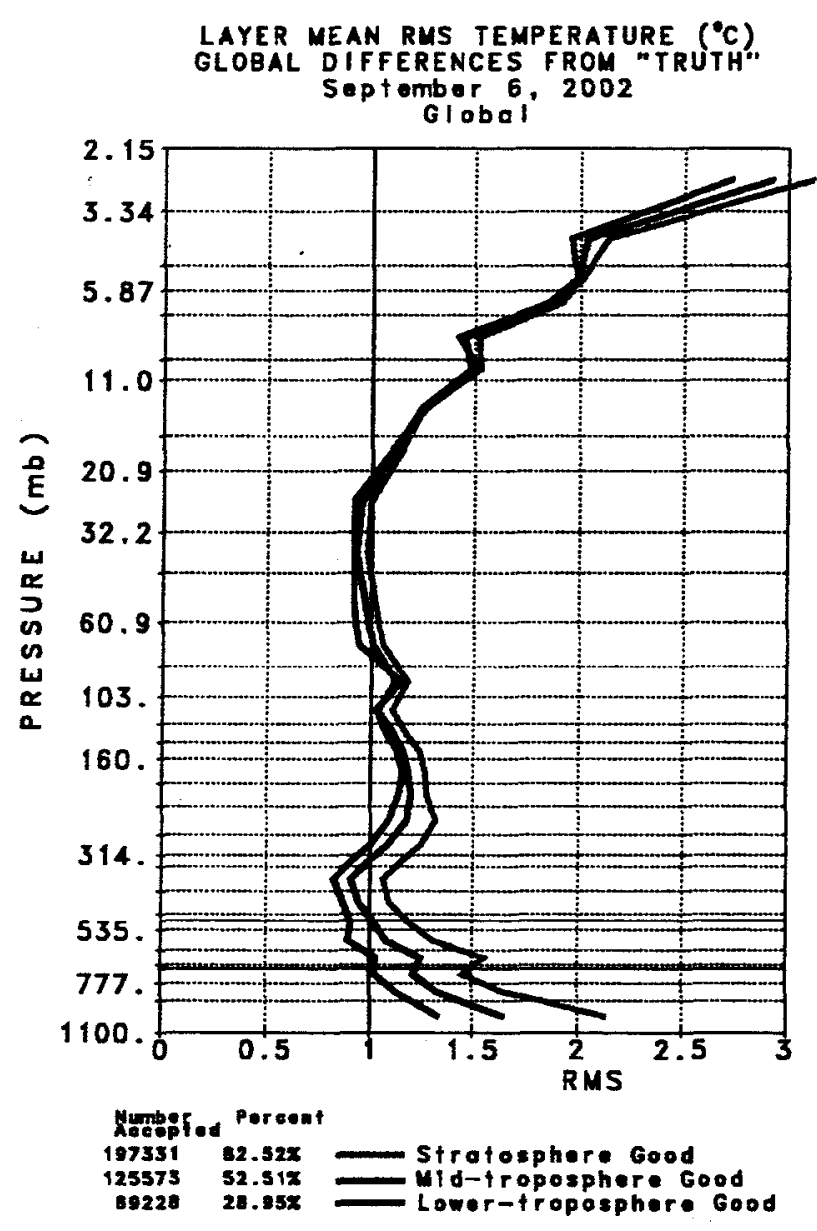

Figure $5 b$ 
Figure 6 shows the RMS difference between retrieved $1 \mathrm{~km}$ tropospheric layer mean temperatures and the collocated ECMWF 3 hour forecast for all accepted cases as a function of retrieved effective cloud fraction. Results are shown for each of the 8 lowest $1 \mathrm{~km}$ layers of the atmosphere. Only those cases passing the appropriate temperature profile test are included in the statistics. Agreement degrades with increasing cioud cover, but only very slowiy. The largest errors are in the 2 lowest layers in the atmosphere, at moderate to high cloud fraction where the percentage acceptance rate is low. RMS temperature differences from ECMWF below $600 \mathrm{mb}$ are somewhat larger than the $1 \mathrm{~K}$ goal for retrieval accuracy. Part of this difference can be attributed to the fact that the ECMWF forecast is not perfect. It is also possible that the accuracy of the ECMWF forecast may be somewhat poorer with increasing cloud cover.

The fundamental parameter used in the determination of geophysical parameters from AIRS/AMSU data is the clear column radiance $\hat{\mathbf{R}}_{i}$, which represents the radiance AIRS channel $i$ "would have seen" if no clouds were in the field of view. Geophysical parameters are determined which are consistent with $\hat{\mathbf{R}}_{\mathrm{i}}$. Derived geophysical parameters whose accuracy degrades slowly with increasing cloud cover implies that the accuracy of $\hat{\mathbf{R}}_{\mathbf{i}}$ also degrades slowly with increasing cloud cover. $\hat{\mathbf{R}}_{\mathrm{i}}$ is an important geophysical parameter derived from AIRS in its own right.

\section{AIRS RMS Temperature Errors vs. Cloud Fraction vs. Effective Cloud Fraction}

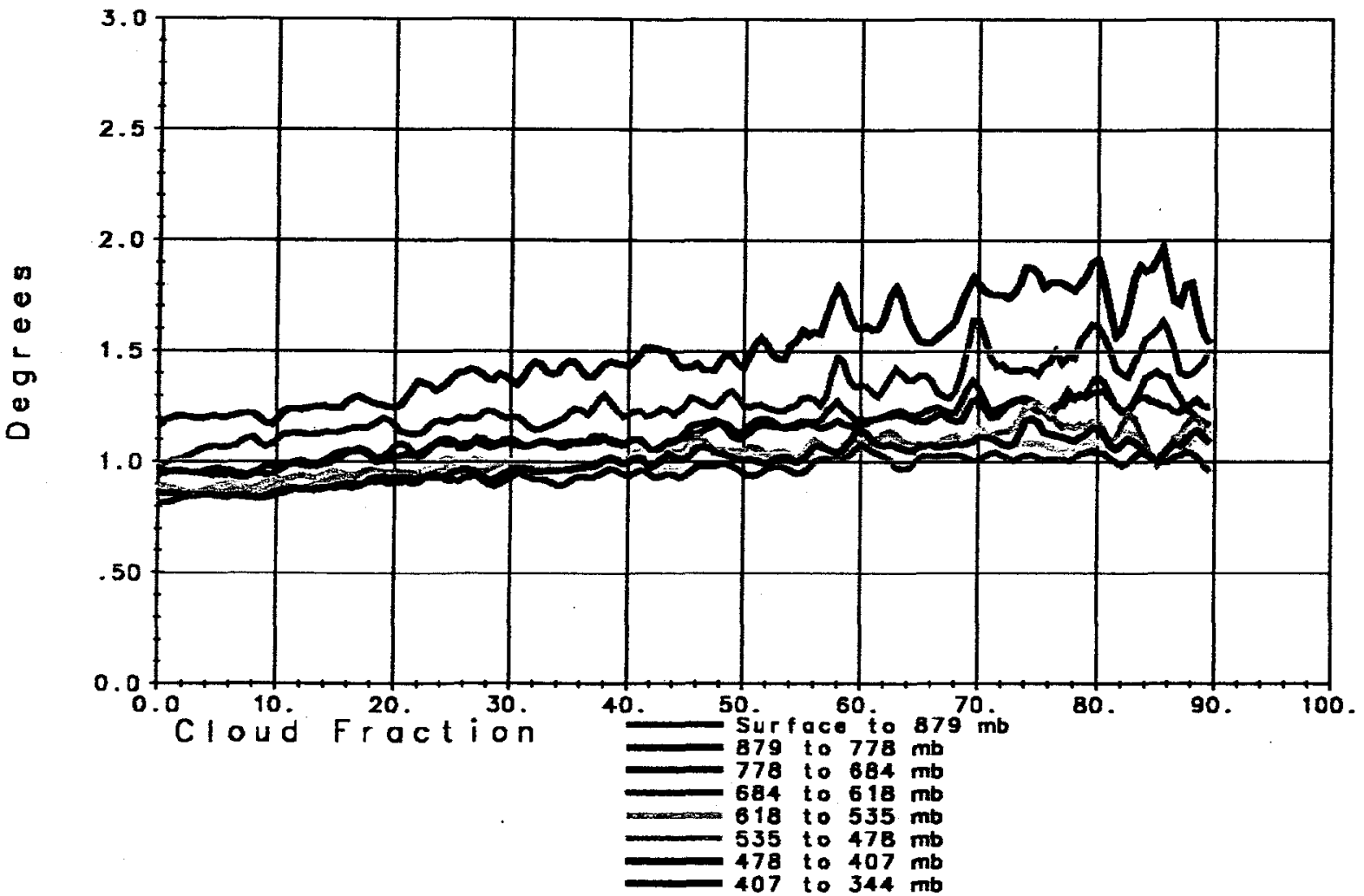

Figure 6

Figure $7 \mathrm{a}$ shows the mean value of $\hat{\mathrm{R}}_{\mathrm{i}}$ (in brightness temperature units) from $650 \mathrm{~cm}-1$ to $756 \mathrm{~cm}^{-1}$ for all non-frozen ocean cases $50^{\circ} \mathrm{N}-50^{\circ} \mathrm{S}$ on September 6, 2002 passing the Tight SST Test. The most opaque portion of the spectrum is near $667.5 \mathrm{~cm}^{-1}$, and is primarily sensitive to atmospheric temperatures near $1 \mathrm{mb}(50 \mathrm{~km})$. Radiances in the surrounding spectral region are also primarily sensitive only to stratospheric temperatures and are not affected by clouds in the field of view. Radiances at frequencies greater than $690 \mathrm{~cm}^{-1}$ see increasing amounts of the troposphere, especially between absorption lines (the locally higher brightness temperatures) and are increasingly affected by cloud cover. Radiances between lines at frequencies higher than $740 \mathrm{~cm}^{-1}$ are also increasingly sensitive to contributions from the ocean surface. 
Figures $7 b$ and $7 c$ show the mean and standard deviation of the differences between $\hat{R}_{i}$ and $R_{i}$ computed from the "truth" for all cases in this geographic domain passing the Tight SST Test, the Loose SST Test, the Lower Tropospheric Temperature Test, and the Mid-Tropospheric Temperature Test, respectively. Figure 7c also contains the channel noise spectrum. In this calculation, the "truth" is taken as the ECMWF forecast of temperature-moisture-ozone profile, along with the ECMWF ocean surface skin temperature. The Masuda Ocean surface emissivity model ${ }^{6}$, revised by Wu and Smith ${ }^{7}$, was used to generate the ocean surface emissivities in the calculation of the expected true radiances. A surface wind speed of 5 meters/sec was used in the emissivity calculation. The surface contribution is the biggest uncertainty in the computation of the "truth" radiances because of errors in both the true ocean skin temperature and in the true surface emissivity.

It is apparent that the difference of clear column radiances from those computed from the truth increases only slightly in the more difficult cloud cases, and in general matches expected radiances to within the AIRS noise level. The increasing difference of clear column radiances from those computed from the "truth" between absorption lines above $740 \mathrm{~cm}^{-1}$ has a large component arising from errors in the "truth".

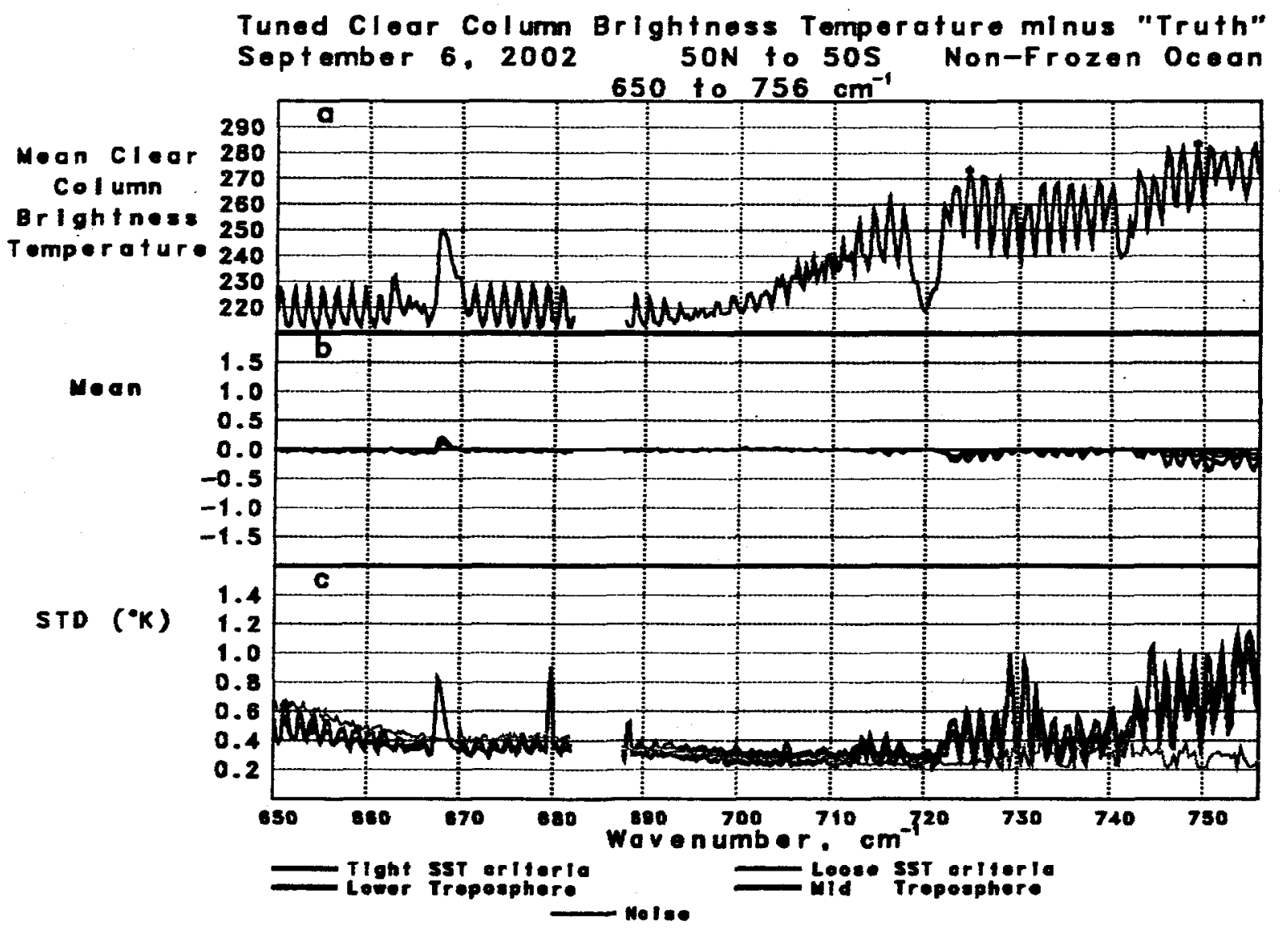

Figure 7

Figure 8 shows histograms of the difference between observed and computed brightness temperatures for the two channels indicated by the black dots in Figure 7, at $724.52 \mathrm{~cm}^{-1}$ and $749.19 \mathrm{~cm}^{-1}$ respectively. These frequencies are primarily sensitive to mid-tropospheric and lower tropospheric temperatures respectively, with a large surface contribution at $749.19 \mathrm{~cm}^{-1}$. Results are shown for the four most stringent quality tests. The differences between the accuracy of clear column radiances at $724.52 \mathrm{~cm}^{-1}$, for cases passing the different quality tests with spatial coverage ranging from $9.12 \%$ to $58.22 \%$, are miniscule, with essentially no outliers in any category. Differences are somewhat larger at $749.19 \mathrm{~cm}^{-1}$ but increase only slightly for cases passing the Mid-Tropospheric Temperature Test. For this reason, all clear column radiances are flagged as good for those cases passing the Mid-Tropospheric Temperature Test. 

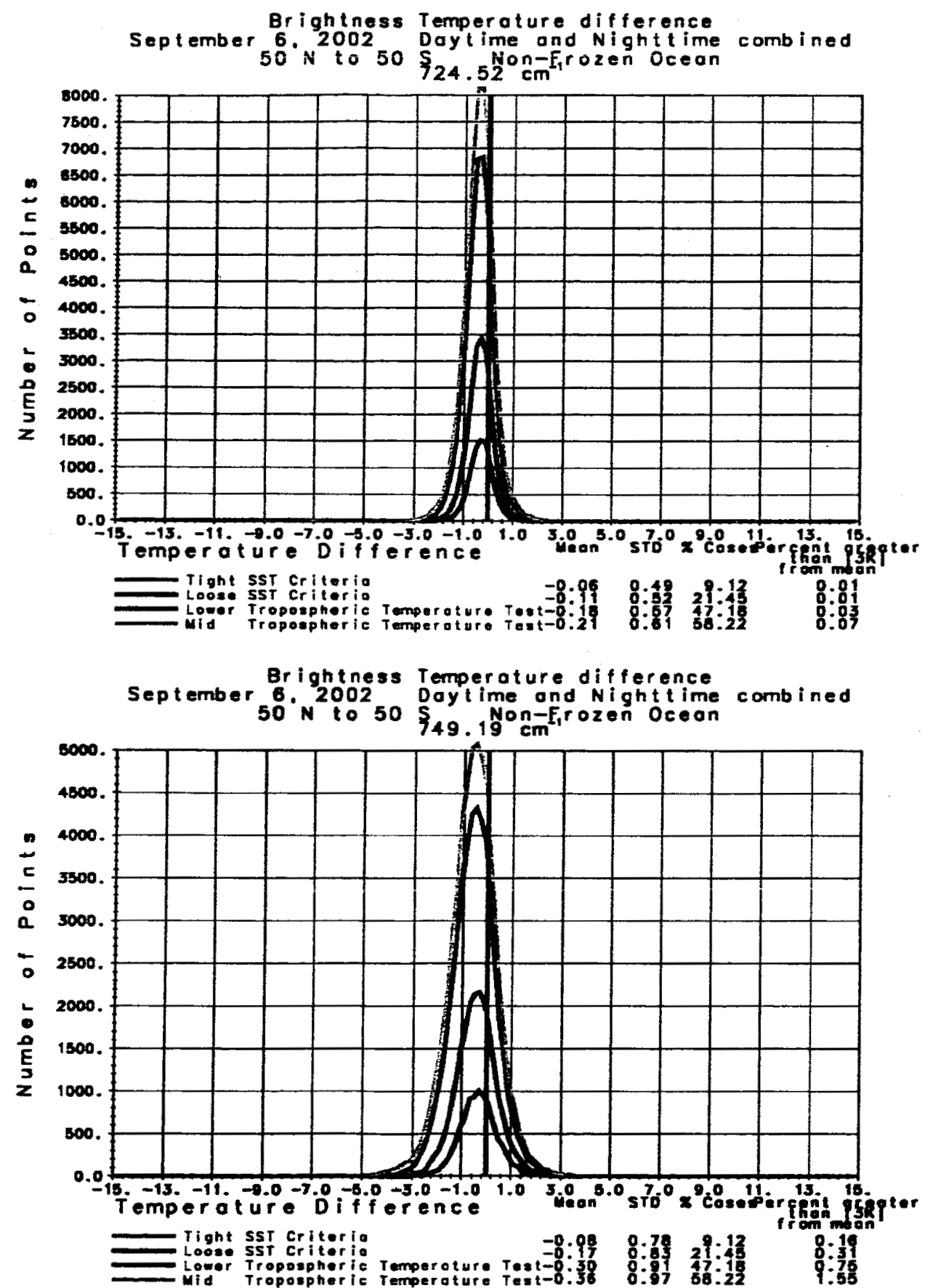

Figure 8

Operational numerical weather prediction centers currently assimilate radiance observations from IR sounder only for those cases thought to be unaffected by clouds. This criterion severely limits the number of $I R$ channel radiances being used in the assimilation processes, and tends to minimize the potential improvement in forecast skill achievable from optimal use of AIRS radiance observations. We encourage operational centers to use the appropriate quality controlled AIRS derived clear column radiances in their assimilation. We use a different approach assimilating AIRS retrieved temperatures directly. All quality controlled temperatures are assimilated. 


\section{FORECAST IMPACT EXPERIMENTS USING VERSION 4.0 AIRS RETRIEVALS}

The data assimilation system used in the experiments is FVSSI which represents a combination of the NASA Finite Volume Generai Circulation Model (FVGCM) ${ }^{8}$ with the NCEP operational Spectral Statistical interpolation (SSI) global analysis scheme implemented at lower than the operational horizontal resolution - T62. The basics of the finite-volume dynamical core formulation are given in DAO's Algorithm Theoretical Basis Document, and the FVGCM has been shown to produce very accurate weather forecasts when run at high resolution ${ }^{5}$. The AIRS temperature profiles produced by SRT were presented to the SSI analysis as rawinsonde profiles with observational error specified at $1^{\circ} \mathrm{K}$ at all vertical levels.

Results are presented for two sets of experiments in which data was assimilated for the period January 1 - January 31 , 2003. Five day forecasts were run every day beginning January 6, 2003 and forecasts every 12 hours were verified against the NCEP analysis, which was taken as "truth". In the first experiment, called "control", all the data used operationally by NCEP was assimilated, but no AIRS data was assimilated. The operational data included all conventional data, TOVS and ATOVS radiances for NOAA-14, 15, and 16, cloud tracked winds, SSM/I total precipitable water and surface wind speed over ocean, QuikScat surface wind speed and direction, and SBUV ozone profiles. In the second experiment, called "all AIRS", temperatures retrieved from all AIRS soundings passing the quality tests described above were assimilated in addition to the data included in the "control" experiment.
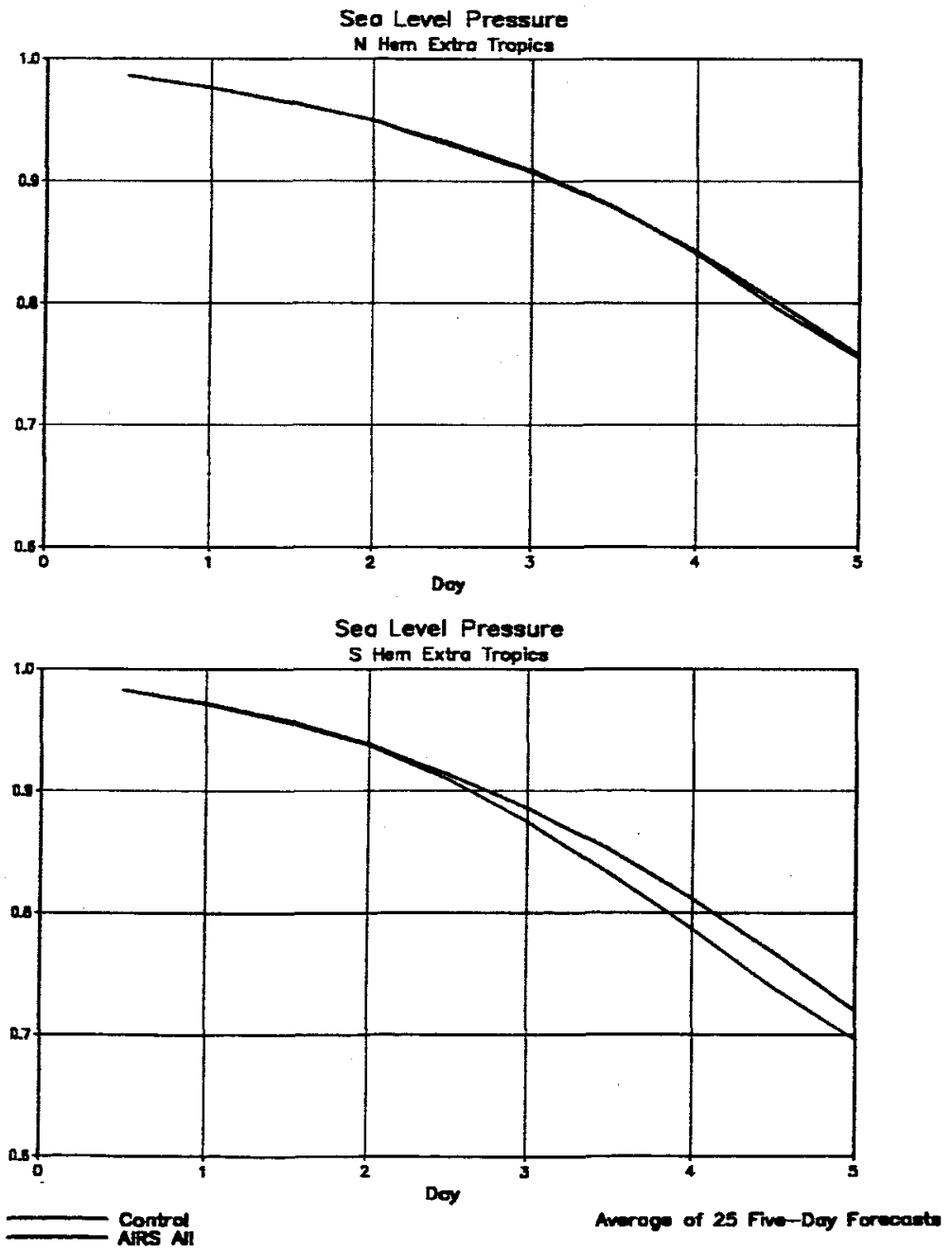

Figure 9 
Figure 9 shows anomaly correction coefficients of forecast sea level pressure verified against the NCEP analysis for both Northern Hemisphere extra-tropics and Southern Hemisphere extra-tropics for both the "control" and "all AIRS" experiments. In the Northern Hemisphere, addition of all AIRS soundings resulted in an improvement in average 5 day forecast skill (a movement of the curve to the right) of the order of 2 hours, and an improvement in 5 day average forecast skill in the Southern Hemisphere more than 6 hours results from assimilation of all AIRS soundings. It should be noted that the Aqua orbit (1:30 ascending) is almost identical to that of NOAA 16 carrying HIRS3, AMSU A and AMSU B, so AIRS/AMSU/HSB soundings are providing additional information to that contained in the AMSU A/AMSU $B$ radiances on NOAA 16 in the same orbit.

A positive forecast impact is a result of a balance between the spatial coverage of the soundings to be assimilated and their intrinsic accuracy. We have shown that the impact of assimilating all AIRS soundings currently flagged as good produces a larger positive input than would be obtained using tighter quality control. We are consequently conducting experiments to further relax the quality control thresholds to find the proper balance to achieve the largest positive impact of the AIRS data on forecast skill.

\section{REFERENCES}

1. Susskind, J., C. D. Barnet, and J. M. Blaisdell, Retrieval of atmospheric and surface parameters from AIRS/AMSU/HSB data in the presence of clouds. IEEE Trans. Geosci. Remote Sensing, 41, 390-409, February 2003.

2. Susskind, J. and R. Atlas, Atmospheric soundings from AIRS/AMSU/HSB. Proc. SPIE Conference, 5425-31, 311319, April 12-15, 2004.

3. Pagano, T. S., H. H. Aumann, D. E. Hagan, and K. Overoye, Prelaunch and in-flight radiometric calibration of the Atmospheric Infrared Sounder (AIRS). LEEE Trans. Geosci. Remote Sensing, 41, 265-273, February 2003.

4. Rosenkranz, P. W., Retrieval of temperature and moisture profiles from AMSU-A and AMSU-B measurements. In Proc. IGARSS, 2000.

5. Goldberg, M. D., Y. Qu, L. M. McMillin, W. Wolff, L. Zhou, and M. Divakaria, AIRS near-real-time products and algorithms in support of operational numerical weather prediction. IEEE Trans. Geosci. Remote Sensing, 41, 379-389, February 2003.

6. Masuda, K., T. Takashima, and Y. Takayama, Emissivity of pure and sea waters for the model sea surface in the infrared window region. Remote Sensing of the Environ., 24, 313-329, 1988.

7. Wu, X. and W. L. Smith, Emissivity of rough sea surface for 8-13 $\mu \mathrm{m}$ : Modeling and verification, Appl. Opt., 36, 2609-2619, 1997.

8. Lin, S. J., A "Vertically Lagrangian" finite-volume dynamical core for global models. Monthly Weather Review, 132, 2293-2307, 2004. 


\title{
Popular Summary
}

\author{
Atmospheric Soundings from AIRS/AMSU in Partial Cloud Cover \\ Joel Susskind and Robert Atlas \\ Laboratory for Atmospheres \\ Earth-Sun Exploration Division
}

This Conference Publication characterizes the performance of the current AIRS Science Team retrieval algorithm, called Version 4.0, and summarizes the differences between Version 4.0 and the pre-launch retrieval algorithm. Results show that accurate geophysical parameters are derived from AIRS/AMSU data in up to $90 \%$ partial cloud cover. Assimilation of all retrieved AIRS atmospheric temperature flagged as good in January 2003 resulted in a modest improvement in forecast skill in the Northern Hemisphere and a very significant improvement in the Southern Hemisphere. Version 4.0 is now being used by the Goddard DAAC to analyze near real time AIRS/AMSU data and will be used to reprocess all previous AIRS/AMSU data starting from September 2002 when the instrument first became stable. 